\title{
Detection, Amplification and Control of Free-Electron Nearfields
}

\author{
Jin-Kyu So ${ }^{1 *}$, Kevin F. MacDonald ${ }^{1}$, and Nikolay I. Zheludev ${ }^{1,2}$ \\ ${ }^{I}$ Optoelectronics Research Centre \& Centre for Photonic Metamaterials, University of Southampton, SO17 1BJ, UK \\ ${ }^{2}$ Centre for disruptive Photonic Technologies, Nanyanag Technological University, Singapore \\ *js1m10@orc.soton.ac.uk
}

\begin{abstract}
We use SNOM-like optical fiber tips functionalized with plasmonic and metamaterial nanostructures to detect, amplify and control the near-field of free electrons in the spectral range from 450 to $850 \mathrm{THz}$.

OCIS codes: (160.3918) Metamaterials; (240.6680) Surface plasmons; (060.4005) Microstructured fibers
\end{abstract}

We experimentally demonstrate that a tapered optical fiber platform, akin to a scanning near-field optical microscopy probe, can be used to interrogate the proximity interaction between free-electron evanescent fields and photonic nanostructures at sub-petahertz optical frequencies. Spectroscopic analysis of far-field emission in the UV/visible wavelength range from tip-mounted nano-grating, thin-film and metamaterial structures (as illustrated in Fig. 1) brought into close proximity with the beam of a scanning electron microscope shows that the evanescent field of moving free electrons can be amplified by a factor of up to 3.4 at $350 \mathrm{~nm}$ by a planar silver film acting as a "poor-man's superlens" for the electron field, and selectively enhanced using resonant periodic nanostructures.
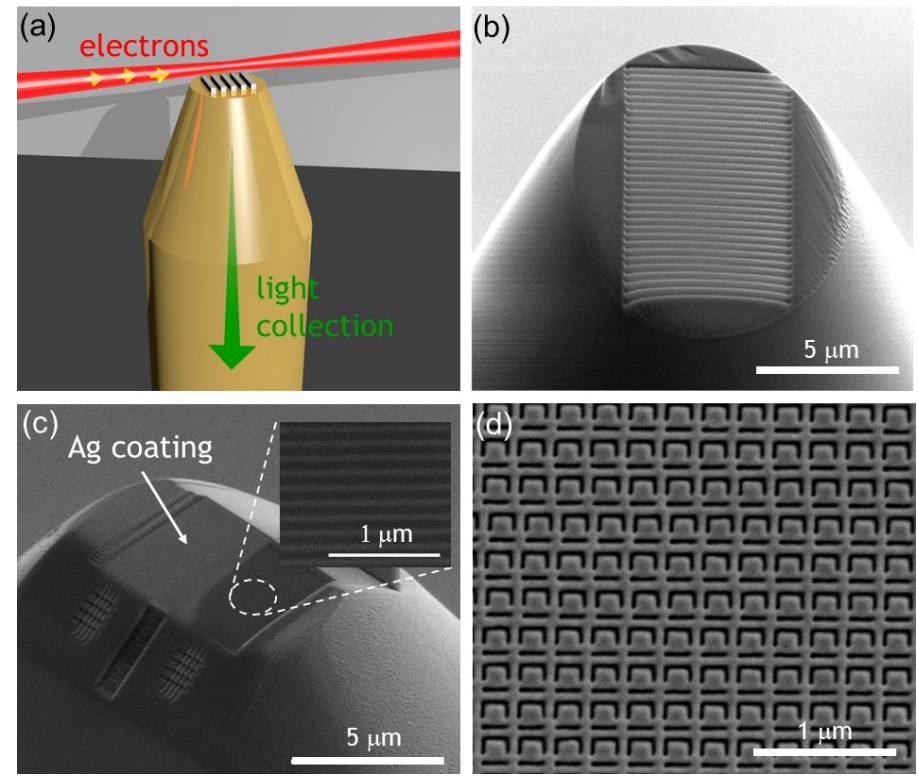

Fig. 1: (a) Artistic impression of an optical fiber probe for electron evanescent fields: light is generated as free electrons pass a the nanostructure on the end facet of a conically tapered, metal-coated fiber tip. Electron microscope images of (b) a $256 \mathrm{~nm}$ period nano-grating on the end facet of a chromium-coated truncated conical fiber probe tip;

(c) a $130 \mathrm{~nm}$ period grating partly coated with a field-amplifying $20 \mathrm{~nm}$ silver film; (d) asymmetric split ring metamaterials fabricated by focused ion beam milling on the end facet of optical fiber tips.

With recent advancements in electron microscopy, spectroscopic techniques based on electron beam are becoming increasingly widely used to interrogate the photonic properties of nanostructures such as photonic crystals, metamaterials, and plasmonic systems. In such applications, free electrons provide unique opportunities for pumping and/or probing nanostructures due to their unrivalled capability for broadband, localized excitation (as compared to optical illumination). While interactions are primarily based on direct electron bombardment of sample structures, it can also be achieved through proximity interactions via the moving electrons' evanescent fields. However, the utility 
of such interactions at optical frequencies is limited by the absence of a standard experimental probe architecture. Here, we present an optical fiber platform which has recently been introduced to facilitate the study of proximity interactions at the nano-scale and discuss the detection, amplification and control of electron fields using fiber tipmounted nano-gratings, plasmonic films and photonic metamaterials.

The optical fiber (Figs. 1a and 1b) plays two major roles in this arrangement, acting both as a tapered substrate for nanophotonic structures and as a light-pipe for the collection of optical radiation generated via proximity interactions. This geometry allows for the luminescence background arising from electrons hitting the substrate material to be minimized and eliminates the need for independent light-collection optics which are typically highly sensitive to the alignment of optical and electron beam foci in three dimensions and can be intrusive to the electron beam path.
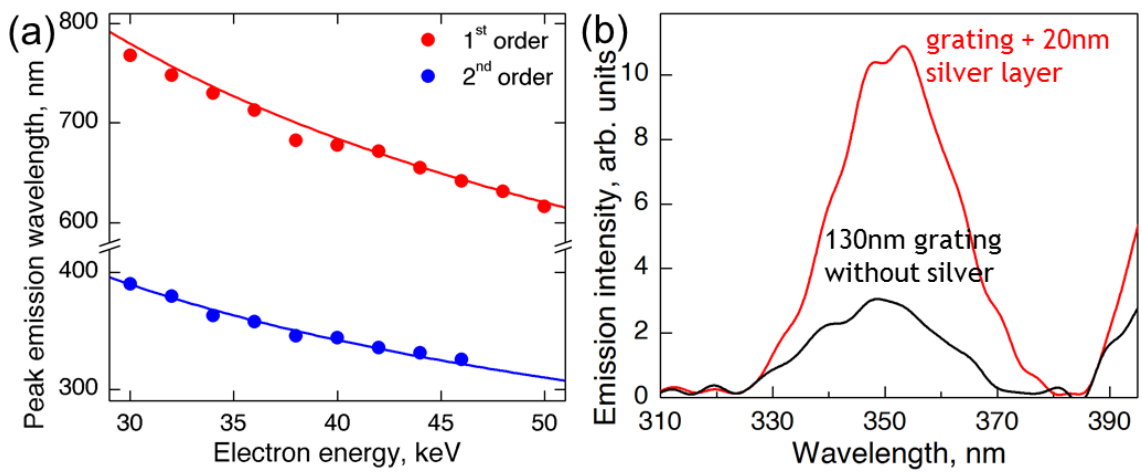

Fig. 2: (a) First- and second-order Smith-Purcell emission wavelengths as a function of electron energy for electrons passing at grazing incidence over a $256 \mathrm{~nm}$ period chromium grating. Data points denote experimental measurements while solid lines derived from the analytical expression for surface-normal Smith-Purcell emission. (b) Electron-beamdriven light emission spectra, at an electron energy of $42 \mathrm{keV}$, from a $130 \mathrm{~nm}$ period grating with (red) and without (black) an intermediate $20 \mathrm{~nm}$ silver film.

Figures 1b-d show a selection of nanophotonic structures studied using the fiber probe configuration. In all cases, the medium-energy (30-50 keV) electron beam of a scanning electron microscope was used: The nano-grating in Fig. $1 \mathrm{~b}$ enables the detection of the evanescent field components of moving free electrons in the 700 to $300 \mathrm{~nm}$ wavelength range via diffractive coupling - the so-called Smith-Purcell effect (Fig. 2a); It is subsequently found that a thin $(\sim 30 \mathrm{~nm})$ silver layer introduced between such a nano-grating and the passing electron beam (Fig. 1c) can amplify the electron field in the same manner as a "poor-man's superlens" amplifies optical evanescent fields - a peak optical emission intensity enhancement factor of 3.4 is achieved at $350 \mathrm{~nm}$ for a $42 \mathrm{keV}$ electron beam (Fig. 2b); Further, we show that a plasmonic metamaterial comprising asymmetric split ring resonators etched by focused ion beam milling into a $50 \mathrm{~nm}$ thick gold film on a fiber tip (Fig. 1d) can resonantly enhance energy coupling from the electron field to propagating optical radiation and can manipulate the relative strengths of diffractive emission orders. In all cases experimental findings are supported by fully three-dimensional finite element and particle-in-cell numerical models. 